\title{
The effect of socioeconomic status, insurance status, and insurance coverage benefits on mortality in critically ill patients admitted to the intensive care unit
}

\author{
Moo Suk Park \\ Division of Pulmonary and Critical Care Medicine, Department of Internal Medicine, Severance Hospital, Yonsei University College of Medicine, Seoul, Korea
}

Socioeconomic status is well known to be related to mortality [1] and is an important health issue in the management of the intensive care unit (ICU) in the United States and Europe [2]. Previous research also shows that the absence of health insurance is associated with a higher mortality rate among patients admitted to the ICU but unadjusted for the severity of illness and data from a single center [3]. Many observational studies have similarly suggested that a lack of insurance is associated with a higher mortality rate, and a systematic review from the American Thoracic Society found that critically ill patients without health insurance receive fewer critical care procedures and show poorer outcomes [3]. Lyon et al. [4] performed a retrospective cohort study using Pennsylvania hospital discharge data, analyzing a total of 138,720 critically ill adults $<64$ years of age treated at 167 acute care hospitals. These authors found that the absence of health insurance is associated with a significant increase in 30day mortality and a decrease in the use of critical care procedures, such as tracheostomy, among critically ill patients using a detailed clinical risk-adjustment protocol. Their study also showed that use of a large multicenter dataset and detailed severity adjustment contributes to the analysis of health outcomes of uninsured critically ill patients [4].

In Europe, two different health care systems exist, specifically the tax-based health care system (THS) and the social health insurance system (SHI). Wernly et al. [5] performed a retrospective post-hoc analysis of data from 16 European countries, analyzing critically ill patients $>80$ years of age admitted to the ICU. They evaluated 4,941 patients with THS and 2,876 with SHI from the previous Very elderly Intensive Patient (VIP)1 and VIP2 studies [5] and found that the associated 30-day mortality rate was similar between both systems; however, patients with SHI were older, sicker, and frailer at baseline. They interpreted their findings as being indicative that a liberal admission policy and an increase in treatment limitations resulted in a trend of ICU excess mortality among patients with SHI [5].

In South Korea, Oh et al. [6] conducted a retrospective observational study of adults aged $>20$ years admitted to the ICU. They included 6,008 patients and found that socioeconomic status was not associated with 30-day mortality in the Korean National Health Insurance (NHI) coverage system. However, the occupation of the patient was associated with 1-year mortality [6]. Meanwhile, although the expansion of Medicaid services for low-income patients has improved mortality in the United States [7], the mortality rate is still higher among critically ill patients with public health insurance coverage only compared to those with ad-

\section{Editorial}

Received: February 13, 2022

Accepted: February 21, 2022

Corresponding author

Moo Suk Park

Division of Pulmonary and Critical

Care Medicine, Department of

Internal Medicine, Severance

Hospital, Yonsei University College of

Medicine, 50-1 Yonsei-ro,

Seodaemun-gu, Seoul 03722, Korea

Tel: +82-2-2228-1955

Fax: +82-2-393-6884

E-mail:pms70@yuhs.ac

Copyright (C) 2022 The Korean Society of Critical Care Medicine

This is an Open Access article distributed under the terms of Creative Attributions Non-Commercial License (https:// creativecommons.org/li-censes/by-nc/4.0/) which permits unrestricted noncommercial use, distribution, and reproduction in any medium, provided the original work is properly cited. 
ditional private health insurance [8].

A financial burden is one of the biggest and most common stress factors among ICU survivors [9]. This burden also impacts their caregivers and is a serious problem. The absence of health insurance coverage is associated with increased mortality among ICU survivors, and a comprehensive approach is required to improve financial coverage for ICU survivors. In a Korean single tertiary hospital cohort study, Cha et al. [10] investigated discharged ICU survivors from 2012 to 2016 and found that low economic status was associated with a higher 1-year mortality rate, even among those patients supported by the NHI coverage system. This study also suggested the necessity of a comprehensive approach to national and regional health care policy for critically ill patients in the ICU and the discharged patients from ICU (ICU survivor) with low income or without insurance coverage [10].

In Yoo et al.'s study [11], the authors performed a single tertiary hospital cohort study that enrolled a total of 515 patients admitted to ICU over 5 years and showed that patients with NHI and additional benefit items experienced greater use of medical resources and improved in-hospital survival. Patients with the three benefit items "cancer," "tuberculosis," and "disability" had lower out-of-pocket medical expenditures due to the implementation of this policy but a higher in-hospital mortality rate [11]. These authors concluded that the Korean NHI benefit extension policy was associated with reduced in-hospital mortality of NHI beneficiaries requiring ventilator care, although the limited nature of a single-center retrospective cohort study requires that ICU care policies be derived from future, larger studies relying on a multicenter database or a big data analysis using Health Insurance Review and Assessment Service or NHI service data.

In conclusion, socioeconomic status, insurance status, and insurance coverage can have beneficial effects on mortality among critically ill patients admitted to the ICU. The national and regional heath care support system is needed with balance between financial burden and management and supportive or end of life care for the critically ill patients and ICU survivors with low income or no insurance coverage.

\section{CONFLICT OF INTEREST}

No potential conflict of interest relevant to this article was reported.

\section{ORCID}

Moo Suk Park

\section{REFERENCES}

1. Kennedy BP, Kawachi I, Glass R, Prothrow-Stith D. Income distribution, socioeconomic status, and self rated health in the United States: multilevel analysis. BMJ 1998;317:917-21.

2. Mackenbach JP, Kunst AE, Cavelaars AE, Groenhof F, Geurts JJ, The EU Working Group on Socioeconomic Inequalities in Health. Socioeconomic inequalities in morbidity and mortality in western Europe. Lancet 1997;349:1655-9.

3. Fowler RA, Noyahr LA, Thornton JD, Pinto R, Kahn JM, Adhikari NK, et al. An official American Thoracic Society systematic review: the association between health insurance status and access, care delivery, and outcomes for patients who are critically ill. Am J Respir Crit Care Med 2010;181:1003-11.

4. Lyon SM, Benson NM, Cooke CR, Iwashyna TJ, Ratcliffe SJ, Kahn JM. The effect of insurance status on mortality and procedural use in critically ill patients. Am J Respir Crit Care Med 2011;184:809-15.

5. Wernly B, Beil M, Bruno RR, Binnebössel S, Kelm M, Sigal S, et al. Provision of critical care for the elderly in Europe: a retrospective comparison of national healthcare frameworks in intensive care units. BMJ Open 2021;11:e046909.

6. Oh TK, Jo J, Jeon YT, Song IA. Impact of socioeconomic status on 30-day and 1-year mortalities after intensive care unit admission in South Korea: a retrospective cohort study. Acute Crit Care 2018;33:230-7.

7. Sommers BD, Baicker K, Epstein AM. Mortality and access to care among adults after state Medicaid expansions. N Engl J Med 2012;367:1025-34.

8. Baldwin MR, Sell JL, Heyden N, Javaid A, Berlin DA, Gonzalez WC, et al. Race, ethnicity, health insurance, and mortality in older survivors of critical illness. Crit Care Med 2017;45:e58391.

9. Khandelwal N, Hough CL, Downey L, Engelberg RA, Carson SS, White DB, et al. Prevalence, risk factors, and outcomes of financial stress in survivors of critical illness. Crit Care Med 2018;46:e530-9.

10. Cha JK, Oh TK, Song IA. Impacts of financial coverage on longterm outcome of intensive care unit survivors in South Korea. Yonsei Med J 2019;60:976-83.

11. Yoo W, Kim S, Kim S, Jeong E, Lee K. Association between the National Health Insurance coverage benefit extension policy and clinical outcomes of ventilated patients: a retrospective study. Acute Crit Care 2022;37:53-60. 